\title{
Mental Health and Actual Impact Brought by COVID-19 Virus Pandemic to Wuhan Citizens
}

\author{
Hanli Xiong ${ }^{1, \mathrm{a}}$
}

\author{
${ }^{1}$ Wuhan Sannew school, Wuhan, Hubei province, China \\ $a^{*}$ Corresponding author. Email: shilishuang@cas-harbour.org
}

\begin{abstract}
Since the COVID-19 virus starts in the year 2020, a mass amount of news records and articles about Wuhan social issues has pumped out. Although most of these articles did show what this pandemic brought to people, there are still some authorized data that seems not realistic. Under this circumstance, the purpose of this research is to fulfill the research gap by collecting Wuhan citizens(who has been staying in Wuhan city since 2020.3 2021.1)'s attitude towards the pandemic, then discover what actual impact did the COVID-19 pandemic isolation brought to Wuhan citizens. The author collected data from 85 Wuhan natives aged from 16 to 60 through a questionnaire, which includes questions about the demographic identity and personal mental state of the testees. The research results are as follows: COVID-19 virus pandemic overall did not affect Wuhan locals in aspects like study, jobs and family environment. In some cases, many of them reported that they even benefited from the quarantine during the pandemic, because their families become more harmonious than before.
\end{abstract}

Keywords: Positive/Negative effects, COVID-19 pandemic, Wuhan

\section{INTRODUCTION}

Citizens' mental state has recently been proven is a vital part of various data report since Wuhan pandemic is now a past and the city is recovering rapidly. However, comparing to the other fundamental data such as GDP and casualty, Wuhan citizens' data in both mental and physical has yet been spread and widely noticed. It is commonly known that people's mental states directly affect their life. To have high working efficiency and well coping in household problems and therefore maintain the whole society functioning in the right course, there is a duty for every scholars or psychology field related people to dedicate themselves on this field. This research was based on a questionnaire designed to investigate Wuhan citizens' (person who has been staying in Wuhan for the past two months) mental states, started from January the 20th in 2021 to February the 25th in 2021. Participants were categorized based to their identities: including gender, age, social identity (student or working), current living situation (live with family or friends, etc.), incomes. Their mental states were analyzed according to whether they has or not infected by COVID-19 virus, the impacts(could be negative or positive) towards their life (e.g. impacts on their job or study and etc.) and their responds on the last part of the questions (question number 14) which tested their mood within the recent two months.

\section{LITERATURE REVIEW}

Unlike other pandemics, COVID-19 brought problems on social sector excepting physical health [1]. Families were facing with a number of immediate concerns, including how to optimally perform remote work without child care, educate their children at home, and prevent disease transmission [2]. Study from Gao Jing(2021)[3] concludes that, if people worry too much about the problems related to COVID-19, they are more likely to get health problems both on physical and psychological among medical personnel, and so as general adults [4]. People tend to feel anxious and unsafe when the environment changes [5]. Thus, in order to fill the defect of people knowing little about the prevalence rate of mental health disorders [6], a questionnaire survey should be taken into account on the research of general citizens' mental state after underwent COVID-19 pandemic. 


\section{METHODS}

\subsection{Research summary}

In order to reach this goal and to get a deeper understanding in of Wuhan living-citizens' mental state, the questionnaire was submitted and was focused on one's career/school study, family circumstance and finally the introspection to his/her own mental state. The background information of the participants can be use to categorize them into different groups and analyze their depression level. Therefore, questions about their background are included in the questionnaire. The research lasted one month and five days, from January the 20th in 2021 to February the 25th in 2021.

The research lasted one month and five days, from January the 20th in 2021 to February the 25th in 2021. The questionnaire was focused on one's career/school study, family circumstance and finally the introspection to his/her own mental state.

The research target is Wuhan living-citizens referred to those people who has been living in Wuhan since March 2020 to January 2021. Questions about where the participants were during the pandemic (from January 2020 to August 2020) were asked, and the report data were canceled if the subject replied a region out of Wuhan.

The questionnaire was separated online and was targeted specific in Wuhan region.

\subsection{Research question}

This questionnaire was constructed by 28 questions in total. The first part of questionnaire tested their basic identity such as age, school grade (including undergraduates)/job field, their incomes per month and what do they know about COVID-19 pandemic. The second part of the questionnaire tested what impacts do they think did the pandemic brought to their study/career. The third part measured the samples' psychological status. In this part of questions, samples were asked to take their recent month as reference in order to answer the question. Furthermore, questions in the third part were divided into two types, a negative type to test their depression level, and a positive type to test their positive feelings, and were random mixed with no sequence in array.

\subsection{Sample's feature description}

Throughout this one month research, 85 questionnaire responses were reserved, and was analyzed. These 85 available data offered by 85 participants were constructed by: 44 female participants (51.76\%) and 41 male participants (48.24\%).

\section{Amount of male/female in 85 participants}

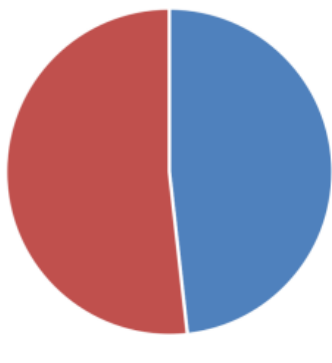

- Male = Female

Figure 1. Amount of male/female in 85 participants (Male and female statistical description).

Participants' age is scattered from 16 to 60 . There are 26 participants(30.59\%) from the age of 16 18; 7 people (8.24\%) from 19 to $22 ; 6$ respondents $(7.06 \%)$ were in the age between 23 24; 17 people $(20 \%)$ were found in the age of $25 \sim 35 ; 10$ participants $(11.76 \%)$ were in the age of 36 44 and finally, the last 19 people $(22.35 \%)$ were divided into the age of $45 \sim 60$ according to their respond.

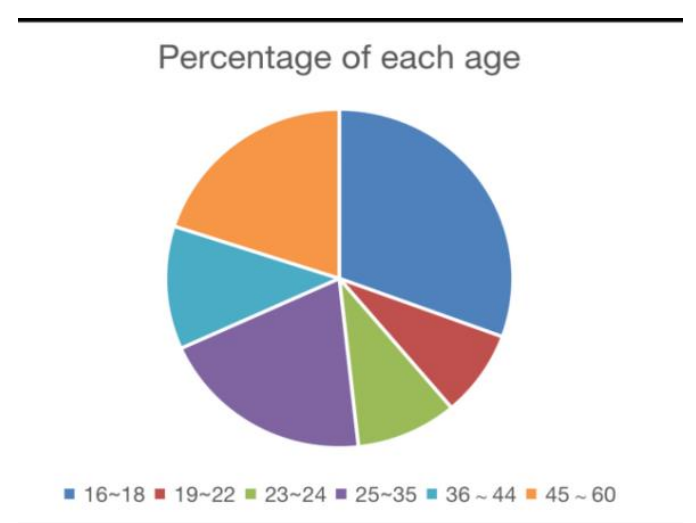

Figure 2. Percentage of each age.

Among those respondents, most of them were "employed" in jobs, accounting for $52.94 \%$ of the total number; "In school" group took the second place, has 32 people, accounting for $37.65 \%$ of the total(figure 3 ). 2 people $(2.35 \%)$ were waiting for employment, 1 person $(1.18 \%)$ is retired. Lastly, 5 people $(5.88 \%)$ were in unemployed statues.

\section{Participants' working status}

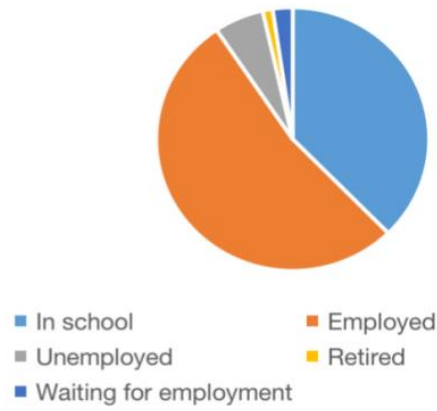

Figure 3. Participants' working status. 
According to the employment subject, there are 6 people received zero income, 2 people gained below 3000RMB, 7 out of the total owned 3000 6000RMB income per month, 15 participants has an income of $6000 \sim 10000 \mathrm{RMB}$ per month, and finally, there are 23people's income stabled above 10000RMB per month(figure 4).

\section{Participants' income status}
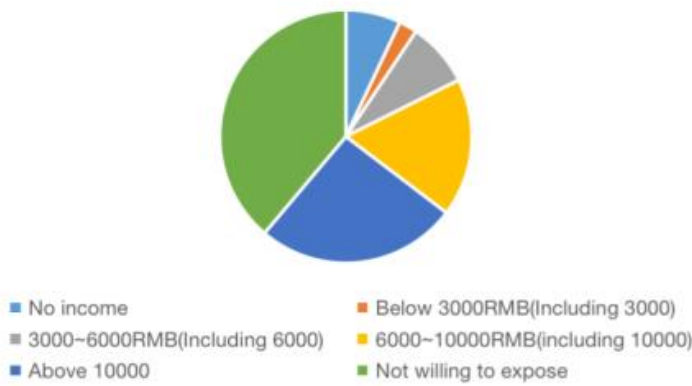

Figure 4. Participants' income status.

\section{RESEARCH RESULT}

\subsection{Impact on Wuhan citizens' obtain employment and job working}

According to the data, there are 51 out of 85 participants who has already obtained employments during the pandemic. From these 51 people, 19 (37.25\%) respondents claim that COVID-19 brought them negative/severe impacts, demonstrate that they are unable to go to their job normally and(or) has a declined job salary. In the contrary, 30(58.83\%) out of 51 people think that their careers were benefited/dramatically enhanced during the pandemic. Enhancements includes: promotion, increase in salary and easier works required, and etc. 2 people says that they were not bothered at all. Therefore, from the data we can conclude that even though the whole city was forced to be lockdown due to the COVID-19 pandemic, half or more than a half of the Wuhan citizens were not bothered by the isolation(figure 5).

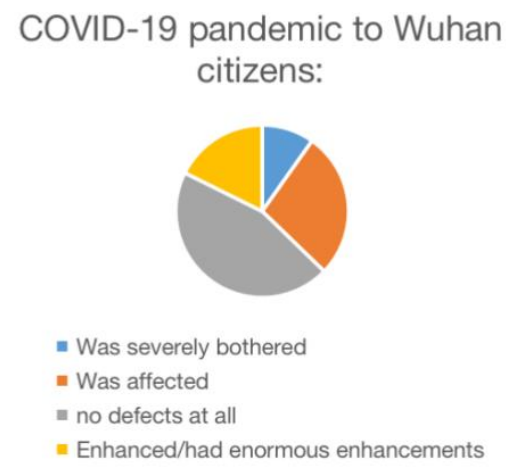

Figure 5. Covid-19 pandemic to Wuhan citizens.

\subsection{Effects from the pandemic to respondents' family relationship}

Among the 85 participants, there are 43 people $(50.59 \%)$ consider that there are neither improvements nor deterioration in their relationship between them and their family; 32 out of 85 people $(37.65 \%)$ think that there is an obvious improvement on the relationship from them to their family, 7 people $(8.24 \%)$ claim that the situation between them and their family faced a deterioration and finally, 3 people $(3.53 \%)$ faced a extreme deterioration among their relationship between our participants and their family(figure 6).

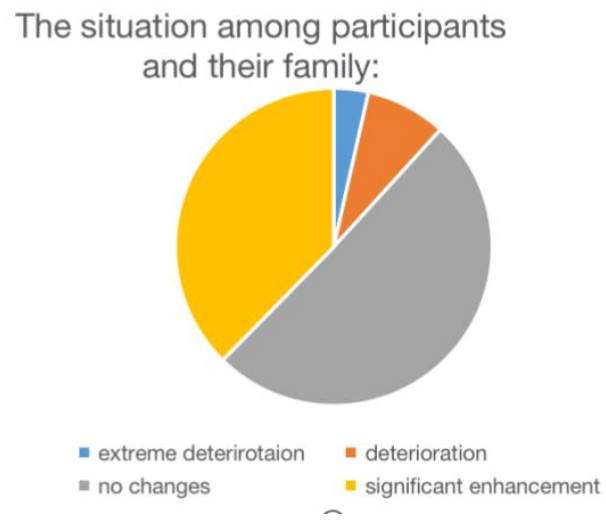

Figure 6. The situation among participants and their family.

\subsection{Effects from the pandemic to respondents' mental state}

This questionnaire provided a section of questions specially designed to test and estimate respondents' mental state. These questions can be divided into two groups: a positive group which focuses on positive emotions, and a negative group which focuses on negative emotions. Each question has a description, and there are five choices for every questions in this section, namely, "Not at all", "Occasionally(<5 times)", "Sometimes(<10 times)", "Many times" and "Always".

The participants were ask to select the choices above according to their mental state within in recent one month.

In the group of questions which focuses on negative emotions, there are $11.23 \%$ and $30.19 \%$ of people selected "Many times" and "Always", whereas in the positive questions, $71.7 \% \sim 75.47 \%$ of the participants chose "Many time" and "Always". Therefore, participants are generally in a healthy mental state.

\section{RESULT ANALYSIS}

After doing research on participants' individual answer sheets, we concluded that there is a positive 
correlation among a participant's job condition, income, mental state and family relationship. The mental state and the family relationship tend to be improved or was stabilized at a high level if there is a enhancement in job or a increase in income, as well as for those participants who end up with low income and(or) deteriorated job condition.

\section{CONCLUSION}

The result was initially anticipated to be dominated by negative data and needs to be fixed by psychological consoling quickly. However, it turns out that more than $70 \%$ of the people felt in a positive way, and most of them had even considerable enhancements in various aspects of their life, which in contrary has significant differences comparing to many similar researches done in other countries. The author feels that this research could be improved by increasing the amount of participants and scattering participants' social classes instead of focus too much on several specific social classes.

\section{REFERENCES}

[1] A. Javed, and M. Afzal. COVID-19 and the Management of Chronic Mental Illnesses. Journal of Psychosocial Rehabilitation and Mental Health, 2020: 1-2.

[2] C.H. Liu, S.N. Doan. Psychosocial Stress Contagion in Children and Families During the COVID-19 Pandemic. Clinical Pediatrics. 2020, 59(9-10): 853-855.

Doi:10.1177/0009922820927044

[3] J. Gao, et al. Impact on physical and mental health among medical personnel in Wuhan during COVID-19 outbreak: a cluster analysis. International Journal of Medical Sciences, 2021, 18(5): 1185-1188.

[4] J. Guo, et al. Coping with COVID-19: Exposure to COVID-19 and Negative Impact on Livelihood Predict Elevated Mental Health Problems in Chinese Adults. International Journal of Environmental Research and Public Health, 2020, 17(11).

[5] K. Usher, J. Durkin, and N. Bhullar. The COVID-19 pandemic and mental health impacts. International Journal of Mental Health Nursing Early View, 2020(1).

[6] D. Liu, et al. Risk Factors Associated with Mental Illness in Hospital Discharged Patients Infected with COVID-19 in Wuhan, China. Psychiatry Research, 2020(292): 113297. 